\title{
High efficiency method to obtain supercoiled DNA with a commercial plasmid purification kit
}

\author{
Antonietta Carbone ${ }^{1}$, Flavia Marialucia Fioretti ${ }^{1}$, Laura Fucci' ${ }^{1}$, Juan Ausió ${ }^{2}$ and \\ Marina Piscopo ${ }^{1 凶}$ \\ 1 University of Naples Federico II, Department of Structural \& Functional Biology, NAPLES, Italy; ${ }^{2}$ University of Victoria, Department of Biochemis- \\ try \& Microbiology, VICTORIA, British Columbia, Canada
}

\begin{abstract}
Supercoiled state corresponds to the active form for plasmid applications. The relaxed circular form of plasmids is often inactive or poorly active. To obtain significant amounts of almost fully supercoiled DNA, we modified the standard protocol of a commercially available Qiagen plasmid purification kit. Our changes led to isolation of almost $100 \%$ of the plasmids in the supercoiled state. The modified protocol was used to purify different plasmids with consistent results. The purified plasmids maintain supercoiled state for about two months. The modified protocol is very advantageous because it allows easy DNA production with high degree of supercoiled form at low cost.
\end{abstract}

Key words: Plasmid DNA, plasmid purification, midi preparation, Qiagen kit, supercoiled plasmid isoform

Received: 19 November, 2011; revised: 11 May, 2012; accepted: 01 June, 2012; available on-line: 11 June, 2012

\section{INTRODUCTION}

Molecular gene manipulation in medicine and molecular biology requires large quantities of highly pure and homogenous plasmid DNA (pDNA) for different applications such as cloning, large scale protein production, DNA vaccination or gene therapy (Anderson et al., 2007). The recent developments in nonviral gene therapy and DNA vaccination have fostered the development of efficient pDNA purification methods. The production and subsequent use of pDNA usually involves several steps, starting with the design of the vector to ensure safety, efficacy and cost-efficient production. The process of isolation and purification of $\mathrm{pDNA}$ involves the disruption of E. coli cells to recover the pDNA, which is then purified using a suitable method. Plasmids are usually produced in an Escherichia coli host by fermentation and prepared using a sequential purification procedure. Plasmids produced and purified in this way are mainly in the supercoiled (sc) form (Prazeres et al., 1998), although conformational changes that occur within the bacterial host or during bacterial processing can induce some single- or double-stranded nicks that result in open circular (oc) or linear (ln) plasmid forms, respectively. The separation of $\mathrm{sc}$ and oc isoforms is one of the key steps in the large-scale purification of $\mathrm{pDNA}$ vectors. In comparison to their viral counterparts, non-viral vectors like naked pDNA are considered to be safer, but they are less effective. The impact of plasmid structure and conformation on transfection efficacy has received some attention in the past. The sc pDNA isoform is the desired topological form since it ensures the most efficient access to the nucleus of the cell and consequently improves gene expression in eukaryotic cells. Moreover, several lines of evidence indicate that high supercoil levels are required for eliciting an effective immune response and, ultimately, protection from infectious challenge (Cupillard et al., 2005). For plasmid DNA therapeutics, the supercoiled plasmid is also desired while the linear and open-circular isoforms are regarded as product impurities (Stadler et al., 2004). Agarose gel electrophoresis (AGE) is the standard method for pDNA isoform separation, with the different isoforms having different mobilities in the gel due to their hydrodynamic differences and the extent of chain entanglement (Doyle et al., 2000). However, purification of pDNA isoforms by AGE is a slow process with low throughput. Furthermore, recovery of DNA from the gel is cumbersome and requires removal of the staining agent used for visualization. According to international regulations, product quality is defined as the percentage of the sc isoform relative to the total pDNA, accordingly, the purity of the sc pDNA must be $100 \%$ with a homogeneity higher than $97 \%$ of the sc isoform from E. coli host (Stadler et al., 2004). This necessity kindled a significant effort within both research and industry to improve the effectiveness of sc pDNA production and purification method (Sousa et al., 2008). For the purification of pDNA several chromatographic methods based on particulate supports have been reported. Besides conventional techniques such as anion exchange, hydrophobic interaction and size exclusion chromatography (Diogo et al., 2005), other methods have been tested with more or less success. With the development of new supports combining different and more specific interactions with pDNA the affinity concept has gained popularity. Based on the natural occurrence of protein-DNA complexes in biological systems and of the favored interactions between particular amino acids and nucleic acid bases (Hoffman et al., 2004; Luscombe et al., 2001), new affinity chromatographic methodology was recently developed that utilizes amino acids immobilized on agarose matrix for the isolation and purification of several nucleic acids (Sousa et al., 2010). Particularly, histidine (Sousa et al., 2005), arginine (Sousa et al., 2008) and lysine (Sousa et al., 2009) have already been used as ligands to efficiently purify sc pDNA. The binding specificity of this isoform to these matrices has been proven to be the result of specific interactions. However, the resolution of the different isoforms in these techniques

$\triangle$ e-mail: e-mail:piscopo@unina.it

Abbreviations: AGE, Agarose gel electrophoresis; In, linear; oc, open circular; pDNA, plasmid DNA; SC, supercoiled; UF, ultrafiltration. 
is often limited and the identification of appropriate buffer conditions can be challenging. Also, the throughput and scalability to large quantities are often problematic. Therefore, there is still a need for new methods of plasmid isoform separation, particularly for large-scale manufacturing of DNA therapeutics. Recently, Latulippe \& Zydney (2011) have demonstrated that transmission of supercoiled plasmid DNA through small pore ultrafiltration (UF) membranes occurs by elongation of the plasmid in the highly converging flow field that forms above the membrane pores providing yet an additional new method of sc pDNA purification. However, most of these methods are time consuming, labor intensive and involve mainly the purification of the sc form from a mixture of the different forms of pDNA. Our research group has, instead, developed a straightforward and inexpensive method that produces highly purified preparations of fully supercoiled pDNA.

\section{MATERIAL AND METHODS}

We performed the plasmid purification modifying the standard protocol of the QIAGEN Plasmid Midi Purification kit (QIAGEN Plasmid Midi Purification handbook, third edition (C) 2005) in two different ways.

For the first approach, the differences with the standard procedure were the following:
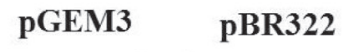

A
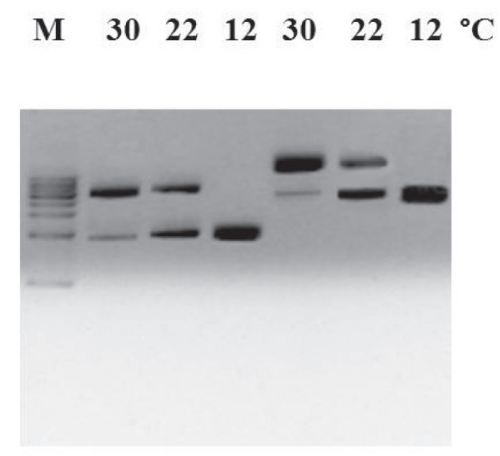

A) the whole procedure for plasmid purification was not carried out at room temperature $\left(15-25^{\circ} \mathrm{C}\right)$, as recommended by the handbook, but in a thermostated room at $12^{\circ} \mathrm{C}$.

All buffers and solvents (except buffer P2 which is the lysis buffer and contains SDS) must be at $4^{\circ} \mathrm{C}$. The purification was carried out in a thermostated room at $12^{\circ} \mathrm{C}$, with some steps performed in a cold room at $4^{\circ} \mathrm{C}$. Numbering of steps is as reported in the Midi Purification handbook: Step 5, buffer P2 is added to bacterial cell suspension; Step 6, buffer P3 is added to the lysate; Step 9, equilibration of the anion-exchange resin in the tips. In addition, the steps corresponding to loading DNA solution onto the resin in the tips, washes and DNA elution (Steps 10, 11 and 12, respectively) were carried out at $4^{\circ} \mathrm{C}$. All the centrifugations were performed at $4^{\circ} \mathrm{C}$. During the procedure of plasmid purification it is important to pay attention to keep the pDNA pellet on ice even during the steps of air drying and resuspension.

The protocol just described will be named as performed at $12^{\circ} \mathrm{C}$ in the following section. Steps 10,11 and 12 have also been performed at $12^{\circ} \mathrm{C}$ giving the same results.

The same protocol modifications were applied to other plasmid purification kits such as Invitrogen Quick Plasmid Midiprep kit and Sigma GenElute HP Plasmid Midiprep Kit.

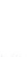

B $\begin{array}{lllll}M & 30 & 22 & 12 & { }^{\circ} \mathrm{C}\end{array}$

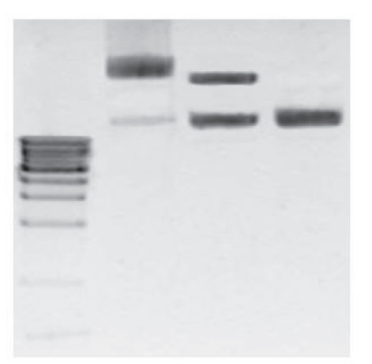

C

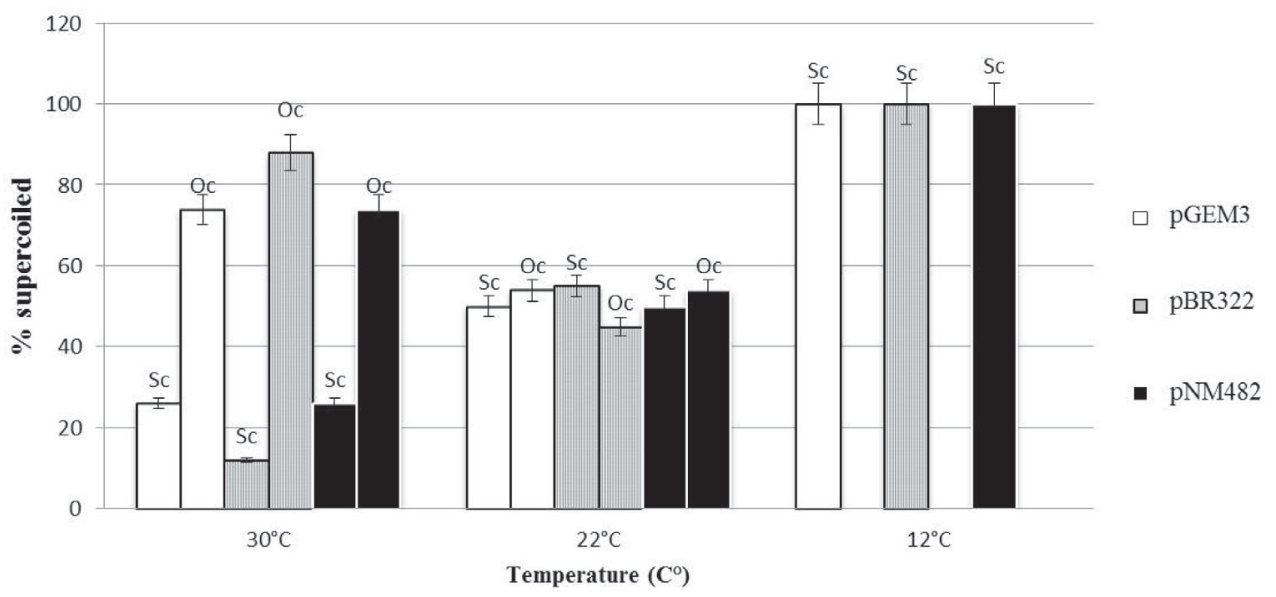

Figure 1. Effect of temperature on supercoiled form yield of different plasmids

Electrophoretic analysis on $1 \%$ agarose gel of pDNA ( $250 \mathrm{ng}$ per lane) purified with the QIAGEN midi preparation kit at 30,22 and $12^{\circ} \mathrm{C}$ (A) pGEM3, pBR322; (B) pNM482; (C) Schematic representation of the plasmid forms obtained by the purification at different temperatures. Quantification of pDNA (pGEM3, pBR322, pNM482) was carried out using a BIO-RAD Quantity One software. (M) 1Kb (Qiagen) marker of molecular weight. 


\section{RT Buffers $4^{\circ} \mathrm{C}$}

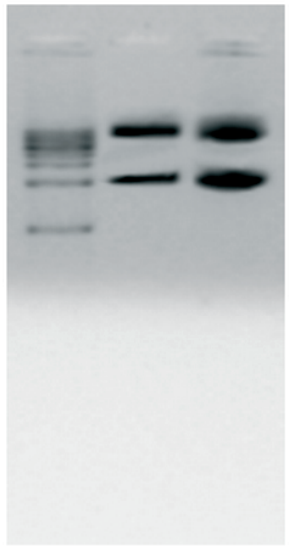

Figure 2. Amount of supercoiled plasmid form obtained by purification at room temperature with or without cold buffers.

Electrophoretic analysis on 1\% agarose gel of pGEM3 plasmid purified at room temperature with the QIAGEN midi preparation kit (lane 2) and at room temperature with buffer stored at $4^{\circ} \mathrm{C}$ (lane 3). (M) $1 \mathrm{~Kb}$ (Qiagen) marker of molecular weight. $250 \mathrm{ng}$ of pDNA was loaded in each lane.

B) In the second approach, all the standard protocol steps of the QIAGEN Plasmid Midi Purification kit were followed with the only difference that all the solvents and buffers (except buffer P2) were maintained at $4^{\circ} \mathrm{C}$ during all the procedure.

Experiments were also performed following the standard procedure (as indicated by the Qiagen Handbook) but at $30^{\circ} \mathrm{C}$ (this procedure will further be named as performed at $\left.30^{\circ} \mathrm{C}\right)$.

The analysis of pDNA was performed by electrophoresis on 1\% agarose gel in TBE buffer. The DNA was visualized by ethidium bromide staining. The samples were analyzed in duplicate on each gel and each experiment was repeated three times. The content of sc and oc

10 days

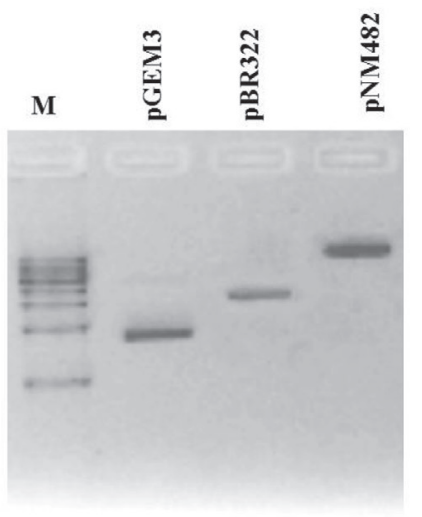

1 month

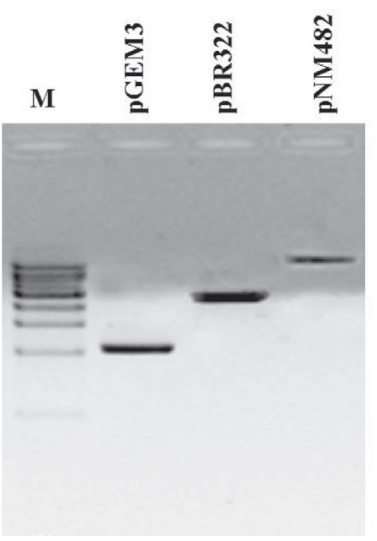

forms were quantified using Gel Doc 1000 system (BioRad) and Quantity One Software.

\section{RESULTS AND DISCUSSION}

Three main requirements have to be met in order to obtain pharmaceutical-grade pDNA at industrial scale. First, a product free from bacterial genomic DNA, RNA, protein and endotoxins has to be obtained. Second, the plasmid has to be in the supercoiled form. Lastly, the process must be cost-effective, scalable and easily amenable to quality control. The aim of this work was to start from a validated protocol for the purification of pDNA from E. coli, in order to easily meet all the requirements indicated above and produce high amounts of pure sc pDNA. We modified the standard protocol of the Qiagen kit for a midi preparation and we tested the new protocol on plasmid DNAs of different length; pGEM3 (2867 bp), pBR322 (4361 bp), pNM482 (8700 $\mathrm{bp})$. The results of midi preparations performed at $12^{\circ} \mathrm{C}$ as described in Materials and Methods were compared with those obtained at $22^{\circ} \mathrm{C}$ as recommended by Qiagen handbook. Midi preparations were also performed at a higher temperature $\left(30^{\circ} \mathrm{C}\right)$ in order to confirm our speculations. Figures $1 \mathrm{~A}$ and $1 \mathrm{~B}$ show results of agarose gel electrophoresis of the three plasmid DNAs purified at different temperatures, as indicated. The results indicate that lowering the temperature produces a progressive increase in the content of the sc form. At $30^{\circ} \mathrm{C}$ pDNAs were obtained mainly in the oc form and a small amount in the sc form; at $22^{\circ} \mathrm{C}$ the sc:oc ratios were about $1: 1$ and at temperatures lower or equal to $12{ }^{\circ} \mathrm{C}$ all pDNAs were in the sc form. Figure $1 \mathrm{C}$ shows a schematic representation of these results. The fraction of the sc form of all used pDNAs increases independently of the length of the plasmid. Also performing the entire procedure of plasmid preparation at $4^{\circ} \mathrm{C}$ (data not shown), we obtained the same results as at $12^{\circ} \mathrm{C}$ but we preferred to perform the experiments at $12^{\circ} \mathrm{C}$ to avoid extended exposure of the researcher to $4^{\circ} \mathrm{C}$.

When the experimental procedure was carried out at room temperature $\left(22^{\circ} \mathrm{C}\right)$ but using buffers and solutions kept at $4^{\circ} \mathrm{C}$ (method $\mathrm{B}$, see Material and Method), the results were similar to those obtained with the standard procedure (as shown in Fig. 2). This result suggests that experiments have to be performed at a temperature not exceeding $12^{\circ} \mathrm{C}$ in order to obtain high amounts of sc pDNA. We tested the modification in temperature also on other plasmid purification kits, as reported in $\mathrm{Ma}$ terial and Methods, and we obtained the same results (data not shown).

We propose that some steps of the procedure are very critical: the step of
Figure 3. Preservation of supercoiled plasmid form after different times of storage at $-20^{\circ} \mathrm{C}$
Electrophoretic analysis on $1 \%$ agarose gel of pDNA (pGEM3, pBR322, pNM482) after 10 days, one month and two months of storage at $-20^{\circ} \mathrm{C}$. (M) $1 \mathrm{~Kb}$ (Qiagen) marker of molecular weight. $250 \mathrm{ng}$ of pDNA was loaded in each lane. 


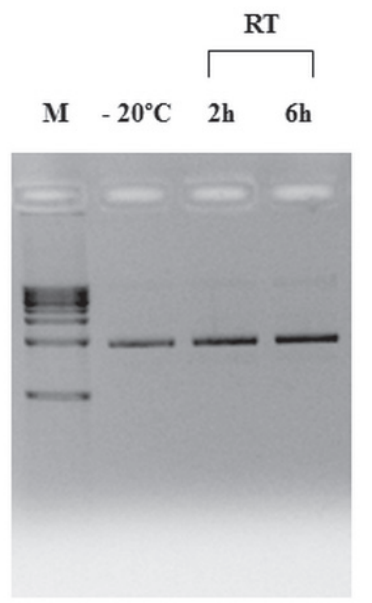

Figure 4. Stability of supercoiled plasmid form after room temperature exposure.

Electrophoretic analysis on 1\% agarose gel of pGEM3 stored at $-20^{\circ} \mathrm{C}$ and after two hours and six hours at room temperature. (M) $1 \mathrm{~Kb}$ (Qiagen) marker of molecular weight. $250 \mathrm{ng}$ of pDNA was loaded in each lane.

DNA adsorption on the resin in the tip by gravity flow, resin washes and DNA elution, because these steps require long time.

Another critical step is the pDNA pellet air drying after isopropanol precipitation and ethanol washes. During these steps we maintain the sample on ice avoiding pipetting DNA, because this may cause shearing.

In our opinion, the low temperature leads to more efficient isolation of sc pDNA because the catalytic efficiency of all enzymatic activities (i.e., nucleases) decreases markedly at lower temperature as reported for e.g., Drosophila topoisomerase II activity at temperatures lower than $20^{\circ} \mathrm{C}$ (Lee et al., 1989; Osheroff et al., 1983). Then a temperature lower than that suggested by the Qiagen kit avoids the production of nicks in the DNA that lead to the production of the open circular plasmid form. We further analyzed the ability of the $\mathrm{pD}$ NAs to retain the sc form. For this purpose, the three plasmids pGEM3, pBR322 and pNM482 obtained in the sc forms were stored at $-20^{\circ} \mathrm{C}$ and analyzed by agarose gel electrophoresis after different times of storage. Usually, a pDNA solution will be stored at this temperature and production of ice crystals may potentially damage the DNA. Figure 3 shows the preservation of the sc form after 10 days and 1 month since the preparation and the production of the oc form in small amount after 2 months of storage.

It is well known that pDNA undergoes conformational changes due to exposure to temperatures above $4^{\circ} \mathrm{C}$. We thus compared the sc form of pGEM3 stored at $-20^{\circ} \mathrm{C}$ with the same pDNA left for two and six hours at room temperature. Figure 4 shows that while the sc form is preserved after two hours, a small amount of the oc form can be observed after six hours at room temperature when compared to the unchanged conformation of pGEM3 stored at $-20^{\circ} \mathrm{C}$.

Finally, we measured the yield of plasmid preparations performed at different temperatures and observed that the temperature did not affect the total yield of the midi preparation, but affected the yield of the sc pDNA.
Until now, plasmid DNA has been used in pharmaceutical development for the production of therapeutic recombinant proteins. Further applications are the use of plasmid vectors for the production of viral particles despite the concerns regarding their potential adverse reactions. Non-viral gene vectors, based on pDNA do not integrate into the genome in contrast to viral vectors nor trigger unintended immunological reactions. The use of pDNA provides an interesting alternative to viral delivery systems because the manufacture, storage and applications of this product pose fewer process and quality control problems. The adoption of this method requires, however, high quantities of pDNA in the sc form. A higher potential for plasmid based gene delivery is expected in genetic vaccination where the expression of a plasmid-coded antigen leads to an immune response in the treated organism. Due to the coiled nature of the circular double helix, plasmid DNA is not necessarily a homogeneous product. In this regard, the method of plasmid purification described here is only an initial step for more complex studies and provides an easy and inexpensive way for obtaining sc pDNA at high quantities for in vitro testing and research and for the development of new transfection techniques.

\section{Acknowledgements}

The authors thank Professor John Finali Pulitzer and Professor Loredana Baccigalupi, University of Naples "Federico II" for providing the pBR322 and pNM482 plasmids.

\section{REFERENCES}

Anderson RJ, Schneider J (2007) Plasmid DNA and viral vector-based vaccines for the treatment of cancer. Vaccine 2: 24-34.

Cupillard L, Juillard V, Latour S, Colombet G, Cachet N, Richard S, Blanchard S, Fischer L, (2005) Impact of plasmid supercoiling on the efficacy of a rabies DNA vaccine to protect cats. Vaccine 23: 1910-1916.

Diogo MM, Queiroz JA, Prazeres DM, (2005) Chromatography of plasmid DNA. J Chromatogr A. 1069: 3-22.

Doyle PS, Ladoux B, Viovy JL, (2000) Dynamics of a tethered polymer in shear flow. Phys Rev Lett 84: 4769-4772.

Hoffman MM, Khrapov, MA, Cox JC, Yao J, Tong L, Ellington AD (2004) AANT: the Amino Acid-Nucleotide Interaction Database. Nucleic Acids Res 32: 174-181.

Latulippe DR, Zydney AL, (2011) Separation of plasmid DNA isoforms by highly converging flow through small membrane pores. J. Colloid Interface Sci 357: 548-553.

Lee MP, Sander M, Hsieh TS, (1989) Single strand DNA cleavage reaction of duplex DNA by Drosophila Topoisomerase II. J Biol Chem 264: 13510-1358.

Luscombe NM, Laskowski RA, Thornton JM, (2001) Amino acid-base interactions: a three-dimensional analysis of protein-DNA interactions at an atomic level. Nucleic Acids Res 29: 2860-2874.

Osheroff N, Shelton ER, Brutlag DL, (1983) DNA topoisomerase II from Drosophila melanogaster. Relaxation of supercoiled DNA. J Biol Chem 258: 9536-9543.

Prazeres DMF, Schluep T, Cooney C, (1998) Preparative purification of supercoiled plasmid DNA using anionexchange chromatography. J Chromatogr A 806: 31-45.

Sousa A, Sousa F, Queiroz JA, (2009) Biorecognition of supercoiled plasmid DNA isoform in lysine-affinity chromatography. J Chromatogr B Analyt Technol Biomed Life Sci 877: 3257-3260.

Sousa A, Sousa F, Queiroz JA, (2010). Differential interactions of plasmid DNA, RNA and genomic DNA with amino acid-based affinity matrices. J Sep Sci 33: 2610-2618.

Sousa F, Prazeres DM, Queiroz JA, (2008) Affinity chromatography approaches to overcome the challenges of purifying plasmid DNA. Trends Biotechnol 26: 518-525.

Sousa F, Tomaz CT, Prazeres DM, Queiroz JA, (2005) Separation of supercoiled and open circular plasmid DNA isoforms by chromatography with a histidine-agarose support. Anal Biochem 343: 183-15.

Stadler J, Lemmens R, Nyhammar T, (2004) Plasmid DNA purification. J Gene Med 1: 54-66. 